\title{
The AF300 and AW922 antibodies against human cytokeratin recognize the cytoskeleton by immunofluorescence in HeLa cells
}

Anna Marchetti

Geneva Antibody Facility, Faculty of Medicine, University of Geneva, 1 rue Michel Servet, CH-1211, Geneva, Switzerland

\begin{abstract}
The AF300 and AW922 antibodies detect by immunofluorescence human cytokeratin in methanolfixed HeLa cells.

\section{Introduction}

Cytokeratins, a diverse family of proteins expressed by different human tissues, are important components of cytoskeletal intermediate filaments (Moll et al., 1982). Two recombinant antibodies targeting human cytokeratin 17 (Uniprot Q04695), AF300 (Lima and Cosson, 2019) and AW922, reveal a cytoskeleton-like pattern in methanol-fixed HeLa cells by immunofluorescence.
\end{abstract}

\section{Materials \& Methods}

Antibodies: ABCD_AF300 and ABCD_AW922 antibodies (https://web.expasy.org/abcd/, ABCD nomenclature) were produced by the Geneva Antibody Facility (https:/www.unige.ch/medecine/antibodies/) as mini-antibodies with the antigen-binding $\mathrm{scFv}$ fused to a rabbit IgG Fc. HEK293 suspension cells (growing in FreeStyle $^{\text {TM }} 293$ Expression Medium, Gibco 12338) were transiently transfected with the vector coding for the scFvFc. Supernatants were collected after 4 days; while AW922 has a high production yield $(80 \mathrm{mg} / \mathrm{L})$, the production yield of AF300 is low $(<5 \mathrm{mg} / \mathrm{L})$.

Antigen: HeLa cells were cultured on a glass coverslip (Menzel-Gläser, 22×22 mm) and grown in DMEM GlutaMAX $^{\mathrm{TM}}$ (Gibco 31966) supplemented with 8\% Fetal Bovine Serum (Gibco 10270).

Protocol: The whole procedure was carried out at room temperature. Cells were rinsed once with PBS, and fixed either with (i) PBS $+4 \%$ paraformaldehyde (w/v) (Applichem A3013) for 30 min, blocked with PBS +40 $\mathrm{mM}$ ammonium chloride $\left(\mathrm{NH}_{4} \mathrm{Cl}\right)$ (Applichem A3661) for $5 \mathrm{~min}$, and then permeabilized in PBS $+0.2 \%$ saponin for $5 \mathrm{~min}$; or (ii) methanol at $-20{ }^{\circ} \mathrm{C}$ for 2 min. Fixed cells were washed once $(5 \mathrm{~min})$ in PBS and once with PBS $+0.2 \%$ $(\mathrm{w} / \mathrm{v})$ BSA (PBS-BSA), and incubated for $30 \mathrm{~min}$ with the primary antibodies (final concentration $5 \mathrm{mg} / \mathrm{L}$ in PBSBSA). After 3 washes (10 min) with PBS-BSA, cells were incubated for $30 \mathrm{~min}$ with secondary goat anti-rabbit IgG conjugated to AlexaFluor-488 (1:400, Molecular Probes A11034). After 3 washes (10 min) with PBS-BSA, cells were mounted on slides (Menzel-Gläser, 76x26 mm) with Möwiol (Hoechst) $+2.5 \%$ (w/v) DABCO (Fluka 33480). Pictures were taken using a Zeiss LSM700 confocal microscope, with a 40x Neofluar oil immersion objective.

\section{Results}

AF300 and AW922 antibodies successfully detect cytoskeletal filaments in HeLa cells (Fig. 1). Side-by-side comparison of cells fixed using different protocols shows that the structure of intermediate filaments is wellpreserved when cells are fixed with methanol, but not when they are fixed with paraformaldehyde (Fig. 1, MetOH vs. PAF). No staining was observed when the primary antibody is omitted (Fig. 1, No Ab). Because of its high production yield, AW922 appears as a good alternative to AF300.

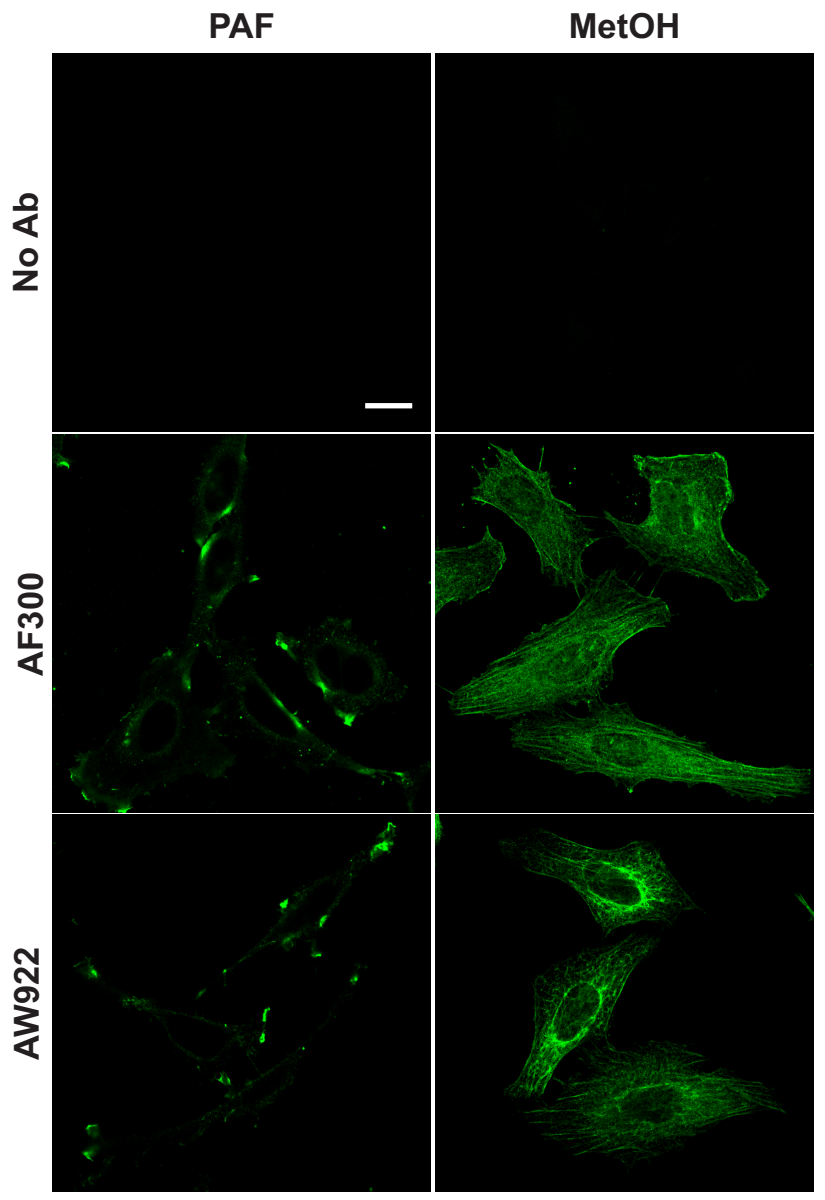

Fig. 1. AF300 and AW922 antibodies successfully label cytoskeletal filaments in HeLa cells (in green). No labelling was seen when the primary antibody was omitted (No Ab panel). PAF: fixation with paraformaldehyde and permeabilization with saponin; MetOH: fixation with methanol. Scale bar: $20 \mu \mathrm{m}$. 


\section{References}

Lima W, Cosson P. The AF161 and AF300 antibodies against human cytokeratin recognize the cytoskeleton by immunofluorescence in HeLa cells. Antibody Reports, 2019, 2:e107. doi:10.22450/ journals/abrep.2019.e107

Moll R, Franke WW, Schiller DL, Geiger B, Krepler R. The catalog of human cytokeratins: patterns of expression in normal epithelia, tumors and cultured cells. Cell. 1982; 31(1):11-24. PMID:6186379.

\section{Conflict of interest}

The authors declare no conflict of interest. 